\title{
Models of gas-grain chemistry in interstellar cloud cores with a stochastic approach to surface chemistry
}

\author{
T. Stantcheva ${ }^{1}$ and E. Herbst ${ }^{2}$ \\ 1 Department of Physics, The Ohio State University, Columbus, OH 43210, USA \\ 2 Departments of Physics, Chemistry, and Astronomy, The Ohio State University, Columbus, OH 43210, USA \\ e-mail: herbst@mps.ohio-state.edu
}

Received 12 March 2004 / Accepted 7 May 2004

\begin{abstract}
We present a gas-grain model of homogeneous cold cloud cores with time-independent physical conditions. In the model, the gas-phase chemistry is treated via rate equations while the diffusive granular chemistry is treated stochastically. The two phases are coupled through accretion and evaporation. A small network of surface reactions accounts for the surface production of the stable molecules water, formaldehyde, methanol, carbon dioxide, ammonia, and methane. The calculations are run for a time of $10^{7}$ years at three different temperatures: $10 \mathrm{~K}, 15 \mathrm{~K}$, and $20 \mathrm{~K}$. The results are compared with those produced in a totally deterministic gas-grain model that utilizes the rate equation method for both the gas-phase and surface chemistry. The results of the different models are in agreement for the abundances of the gaseous species except for later times when the surface chemistry begins to affect the gas. The agreement for the surface species, however, is somewhat mixed. The average abundances of highly reactive surface species can be orders of magnitude larger in the stochastic-deterministic model than in the purely deterministic one. For non-reactive species, the results of the models can disagree strongly at early times, but agree to well within an order of magnitude at later times for most molecules. Strong exceptions occur for $\mathrm{CO}$ and $\mathrm{H}_{2} \mathrm{CO}$ at $10 \mathrm{~K}$, and for $\mathrm{CO}_{2}$ at $20 \mathrm{~K}$. The agreement seems to be best at a temperature of $15 \mathrm{~K}$. As opposed to the use of the normal rate equation method of surface chemistry, the modified rate method is in significantly better agreement with the stochastic-deterministic approach. Comparison with observations of molecular ices in dense clouds shows mixed agreement.
\end{abstract}

Key words. ISM: abundances - ISM: molecules - molecular processes

\section{Introduction}

A standard approach to modeling the chemistry in the interstellar medium has been to use the rate equation method (Pickles \& Williams 1977; Hasegawa et al. 1992). In this method, the rate at which the abundance of a chemical species changes is governed by terms that contain the products of the abundances of the chemical species that react to produce and destroy it; the coefficients multiplying each term are known as rate coefficients. The basic assumption in such an approach is that the process being simulated is deterministic; that is, at any given time the evolution of the system is completely determined and can be calculated given the initial conditions. While the method has been applied successfully to gas-phase chemistry (Herbst \& Klemperer 1973; Prasad \& Huntress 1980; Terzieva \& Herbst 1998; Roberts et al. 2002), its success for granular chemistry has been somewhat questionable. In the work of Tielens \& Hagen (1982) and Charnley et al. (1997), and more especially in an unpublished talk given by Tielens in 1995, attention was focused on the fact that the rate equation method uses average chemical abundances and is valid only as long as the actual abundances can be safely replaced with some average values.
Furthermore, it was argued that in conditions where reactions occur much faster than the rate at which species accrete onto grain surfaces (the so-called "accretion limit"), the abundances of some reactants will be so low (one or fewer particles per grain) that it becomes questionable to consider them as averages. In order to avoid this problem, Tielens \& Hagen (1982) used an alternative method based on a Monte Carlo algorithm that follows the order of individual particles as they land on a grain. While such an approach takes some consideration of the randomness of chemical processes, it is not completely stochastic. A rigorous stochastic model of interstellar surface chemistry was first developed by Charnley (2001), in which the so-called "chemical master equation" was solved by means of Monte Carlo simulations in the fashion prescribed by Gillespie (1976, 1992). Charnley (1998) had earlier developed a similar stochastic method for gas-phase chemistry.

While the advantage of the stochastic approach over the rate equation method is clear - the former takes into consideration the statistical nature of grain chemistry and preserves information about the fluctuations in the surface abundances - the stochastic approach has some major drawbacks. First, its solution requires orders-of-magnitude more computing time than 
the rate equation method. In addition, there are problems with the resolution if one tries to apply it to gas-grain simulations. With a fractional grain density of $10^{-12}$ with respect to hydrogen, if the dust grains are to be resolved the resolution must be equal to or better than $10^{-12}$. This requirement has so far been proven virtually prohibitive for such calculations. If, on the other hand, one tries to use Monte Carlo simulations for the surface chemistry only, while using rate equations for the gas-phase chemistry, many questions arise as to how the methods can be incorporated together. To our knowledge, such an incorporation has not been successfully accomplished. In its absence, a semi-empirical method, in which the rate equations are modified to mimic the results of stochastic calculations, has proven useful (Caselli et al. 1998, 2002; Stantcheva et al. 2001).

There is another method, however, by which the master equation can be solved, which allows the coupling of the stochastic and the deterministic approaches within a single framework. In particular, it has been shown by two groups, independently of each other, that the master equation for diffusive surface chemistry can be solved by direct numerical integration (Green et al. 2001; Biham et al. 2001). We have previously extended this direct approach for use in time-dependent models of granular chemistry leading to the formation of methanol and its isotopomers (Stantcheva et al. 2002; Stantcheva \& Herbst 2002). The details of the mathematics can be found in Stantcheva et al. (2002). The advantage of the direct master equation method is that while it is still based on the stochastic approach, its implementation is very similar to that used in the rate equation method. Both procedures lead to systems of coupled first-order differential equations, so that incorporating them together in a model becomes a relatively straightforward procedure with the addition of adsorption and desorption terms.

In this work, we report our success in incorporating for the first time the rate equation and direct master equation methods into a gas-grain model of a quiescent interstellar cloud core. This method will be labeled the "stochastic-deterministic" or "master equation" approach, with the former term referring to the fact that we treat the surface chemistry in a stochastic manner and the gas-phase chemistry deterministically. The remainder of the paper is organized as follows. An overview of the master equation approach and its implementation is given in Sect. 2. The chemical model is described in Sect. 3, while the results are given in Sect. 4 and compared with the rate equation approach. Comparisons with observations and other models are discussed in Sect. 5, while Sect. 6 contains a discussion of our results.

\section{Theory}

As in earlier papers concerning the use of the direct master equation treatment (Green et al. 2001; Biham et al. 2001; Stantcheva et al. 2002; Stantcheva \& Herbst 2002; Lipshtat et al. 2004) the chemistry occurring on the grains is here limited to diffusive processes involving weakly-bound species, a mechanism known as the Langmuir-Hinshelwood approach. A possible extension to other mechanisms of surface chemistry, such as the Eley-Rideal and physisorption-chemisorption approaches (Cazaux \& Tielens 2002), is currently under consideration.

The master equation approach is built upon a probabilistic framework that takes into account the fact that chemical processes are statistical in nature. The system is characterized at some initial time by a state, which is defined by the abundances of the chemical species. As time progresses, different states can be accessed. In the case of granular surfaces, each state represents a different set of numbers of abundances (e.g., 1 hydrogen atom, two oxygen atoms, etc.), and is associated with a probability for the system to be in that particular state. The master equation describes the temporal evolution of these probabilities, and its solution gives the probability for the system to be in each of the possible states at any given time. The average abundances of individual species, as well as any fluctuations in the abundances or in the correlations between the abundances of two or more species, can be calculated from the state probabilities by using well known statistical formulae (Stantcheva et al. 2002).

The master equation in its analytical form contains information about all possible states of the system, which in general are infinite in number. In order for the equation to be solved numerically, however, it is necessary that this number be limited to a reasonable size. For that purpose we have introduced two approximations (Stantcheva et al. 2002; Stantcheva \& Herbst 2002). First, we divide the surface chemical system into two sub-systems: one of them containing a set of surface species selected in advance called "probabilistic" or "stochastic", and the other containing the remaining neutral species. The probabilistic species, typically atoms and radicals, are those with very low average abundances caused by high reactivity with other species. The other species have sufficiently high surface abundances that they need not be treated by a stochastic method; indeed, it is not possible to include them in such an approach given the very large amount of computing time that would be needed. The stochastic approach developed here is essentially useful only for species with average surface abundances of unity or less, whereas the normal unreactive species can reach abundances of one monolayer or more. Because such a division requires advance knowledge of surface abundances, when in doubt we use the results produced in deterministic models, in which all the chemistry is simulated via rate equations. While the results of such models can be questionable concerning some of the surface species, they can be used as a good starting point to give a general idea of which species are probabilistic.

Once the species are separated into two sets, the possible states of the chemical system are then determined only by the abundances of the surface probabilistic species, and only they are included in the master equation. In particular, out of the total 21 surface species that participate in diffusive reactions in our current model network, the probabilistic species are $\mathrm{H}$, $\mathrm{O}, \mathrm{OH}, \mathrm{HCO}, \mathrm{H}_{3} \mathrm{CO}, \mathrm{C}, \mathrm{CH}, \mathrm{CH}_{2}, \mathrm{CH}_{3}, \mathrm{~N}, \mathrm{NH}$, and $\mathrm{NH}_{2}$. With these species, the master equation is then decomposed to a set of first order differential equations for the 12-body joint probabilities of the states determined by the abundances of these species. From these joint probabilities, information about the individual surface probabilistic species can be obtained. 
The remaining 9 "normal" surface species are $\mathrm{H}_{2}, \mathrm{O}_{2}, \mathrm{H}_{2} \mathrm{O}$, $\mathrm{CO}, \mathrm{H}_{2} \mathrm{CO}, \mathrm{CH}_{3} \mathrm{OH}, \mathrm{CO}_{2}, \mathrm{CH}_{4}$, and $\mathrm{NH}_{3}$, and their abundances are calculated via equations analogous to but slightly distinct from standard rate equations (Stantcheva et al. 2002).

The second very important approximation is to limit our consideration to only those states in which the abundance of each probabilistic species does not exceed a certain number. For that purpose we choose a set of cutoff values $\mathcal{N}=$ $\left\{\mathcal{N}_{1}, \mathcal{N}_{2}, \ldots\right\}$ so that we ignore any state for which the abundance of the $i$ th species is larger than the corresponding limit $\mathcal{N}_{i}$. In addition, we neglect any states for which the total number of all species exceeds a given number $\mathcal{N}_{\text {tot }}$. In coding this constraint, one must observe caution. Even though in the final result only a few states will have a significant probability, numerical integration can easily lead to accumulation of error. For example, if by neglecting certain unlikely states, the sum of the probabilities becomes equal to 0.99 instead of unity, as it must be if all states are considered, in only 10 integration steps this sum will have dropped to 0.9 and the cut-off error will be at the $10 \%$ level. In order to avoid such an accumulation of error, at each step we evaluate the total probability, and then the probability for the state with no surface abundances is increased by such an amount as to compensate for the loss due to the neglected high-abundance states. While such a procedure decreases the numerical error significantly and is justified by the fact that the state of zero abundance of the "probabilistic" species is typically by far the most likely in the accretion limit, there is still some inaccuracy remaining. Note also that this technique makes the abundances of individual probabilistic species formal lower limits to the true abundances. A simple way to determine the magnitude of the inaccuracy is to run the stochastic-deterministic model with increasing sets of values for the cutoffs $\mathcal{N}_{i}$ 's and $\mathcal{N}_{\text {tot }}$. If the results do not converge, then a new set of higher cutoff values is chosen and the model run again. If convergence still does not occur, then some species must be removed from the list of probabilistic molecules.

As long as the calculated abundances for probabilistic species correspond to significantly less than one particle per grain, our calculations show that the cut-off error can be overcome. In all cases reported here, cutoffs for the probabilistic species of two and a total limit of such particles equal to four are sufficient to produce results with reasonable accuracy. With such cutoffs, each run took an hour or less of computing time on the SV1 machine of the Ohio Supercomputer Center. A problem will arise when one or more species begins to have an abundance corresponding to about one particle per grain. In such a regime, the rate equation method is still not entirely valid, yet the cut-off error in the master equation approach becomes significant and higher cutoffs are required.

Some additional comments on numerical accuracy are made in the appendix.

\section{Model}

The gas-phase network is based on a modified version of the so-called New Standard Model, as developed and described in Bettens et al. (1995) and Terzieva \& Herbst (1998). In this network, 455 atoms and molecules in the gas, both neutral
Table 1. Initial fractional abundances.

\begin{tabular}{lr}
\hline \hline $\begin{array}{l}\text { Element } \\
\text { (initial form) }\end{array}$ & $\begin{array}{r}\text { Fractional } \\
\text { abundance }\end{array}$ \\
\hline $\mathrm{He}$ & $1.4(-01)$ \\
$\mathrm{N}$ & $2.14(-05)$ \\
$\mathrm{O}$ & $1.76(-04)$ \\
$\mathrm{H}$ & $5.0(-05)$ \\
$\mathrm{H}_{2}$ & $0.50(-00)$ \\
$\mathrm{C}^{+}$ & $7.3(-05)$ \\
$\mathrm{S}^{+}$ & $8.0(-08)$ \\
$\mathrm{Si}^{+}$ & $8.0(-09)$ \\
$\mathrm{Fe}^{+}$ & $3.0(-09)$ \\
$\mathrm{Na}^{+}$ & $2.0(-09)$ \\
$\mathrm{Mg}^{+}$ & $7.0(-09)$ \\
$\mathrm{e}$ & $7.3(-05)$ \\
$\mathrm{P}^{+}$ & $3.0(-09)$ \\
$\mathrm{Cl}^{+}$ & $4.0(-09)$ \\
\hline
\end{tabular}

and charged, react via 4218 reactions. The conditions chosen for study pertain to quiescent cloud cores, in which the hydrogen is mainly molecular and at a density of $10^{4} \mathrm{~cm}^{-3}$ $\left(n_{\mathrm{H}}=2 \times 10^{4} \mathrm{~cm}^{-3}\right)$. We have in the main used a temperature of $10 \mathrm{~K}$, but also present results for $15 \mathrm{~K}$ and $20 \mathrm{~K}$. The initial gas-phase fractional abundances are shown in Table 1; these are based on so-called "low-metal" elemental abundances. A cosmic ray ionization rate $\zeta\left(\mathrm{s}^{-1}\right)$ of $1.3 \times 10^{-17}$ has been utilized.

Although all gas-phase neutral species are allowed to adsorb onto grains and desorb from them thermally and via cosmic-ray-induced heating (Hasegawa \& Herbst 1993), only 21 species are assumed to be involved in active surface chemistry. The idea is not to be complete, but to include the major processes leading to the dominant mantle species and to test the stochastic-deterministic method. A list of the included surface reactions is given in Table 2, which consists of the reactions considered in Stantcheva et al. (2002) plus seven new reactions to account for the production of methane and ammonia. We have chosen to keep the reaction sequence for production of methanol via successive hydrogenation of $\mathrm{CO}$ and subsequently formaldehyde despite the existence of ambiguous laboratory data (Hiraoka et al. 2000; Watanabe \& Kouchi 2002).

The neutral species from the gas can accrete onto the dust grains or desorb at rates calculated in the fashion given in Hasegawa et al. (1992) and Hasegawa \& Herbst (1993). We have considered only weakly bound (physisorbed) species that can sweep across the grain surface via the LangmuirHinshelwood diffusion mechanism by thermal hopping between the binding sites, or, in the case of hydrogen atoms only, by quantum mechanical tunneling (Hasegawa et al. 1992; Cazaux \& Tielens 2002). The energy barriers against diffusion $\left(E_{\mathrm{b}}\right)$ and the binding energies $\left(E_{\mathrm{D}}\right)$ for reactive surface species are given in Table 3. Accretion, evaporation, cosmic ray-induced desorption, and diffusion rates for the atoms $\mathrm{H}$, $\mathrm{O}, \mathrm{C}$, and $\mathrm{N}$ are shown for $10 \mathrm{~K}, 15 \mathrm{~K}$, and $20 \mathrm{~K}$ in Table 4 . The diffusion rates in the model are obtained in the manner 
Table 2. Surface reactions in the model.

\begin{tabular}{lll}
\hline \hline Number & Reaction & $E_{\mathrm{a}}(\mathrm{K})$ \\
\hline 1 & $\mathrm{H}+\mathrm{H} \longrightarrow \mathrm{H}_{2}$ & \\
2 & $\mathrm{H}+\mathrm{O} \longrightarrow \mathrm{OH}$ & \\
3 & $\mathrm{H}+\mathrm{OH} \longrightarrow \mathrm{H}_{2} \mathrm{O}$ & \\
4 & $\mathrm{H}+\mathrm{CO} \longrightarrow \mathrm{HCO}$ & 2500 \\
5 & $\mathrm{H}+\mathrm{HCO} \longrightarrow \mathrm{H}_{2} \mathrm{CO}$ & \\
6 & $\mathrm{H}+\mathrm{H}_{2} \mathrm{CO} \longrightarrow \mathrm{H}_{3} \mathrm{CO}$ & 2500 \\
7 & $\mathrm{H}+\mathrm{H}{ }_{3} \mathrm{CO} \longrightarrow \mathrm{CH}_{3} \mathrm{OH}$ & \\
8 & $\mathrm{H}+\mathrm{C} \longrightarrow \mathrm{CH}$ & \\
9 & $\mathrm{H}+\mathrm{CH} \longrightarrow \mathrm{CH}_{2}$ & \\
10 & $\mathrm{H}+\mathrm{CH} \longrightarrow \mathrm{CH}_{3}$ & \\
11 & $\mathrm{H}+\mathrm{CH} \longrightarrow \mathrm{CH}_{4}$ & \\
12 & $\mathrm{H}+\mathrm{N} \longrightarrow \mathrm{NH}$ & \\
13 & $\mathrm{H}+\mathrm{NH} \longrightarrow \mathrm{NH}_{2}$ & \\
14 & $\mathrm{H}+\mathrm{NH} \longrightarrow \mathrm{NH}_{3}$ & \\
15 & $\mathrm{O}+\mathrm{O} \longrightarrow \mathrm{O}_{2}$ & \\
16 & $\mathrm{O}+\mathrm{CO} \longrightarrow \mathrm{CO}_{2}$ & 1000 \\
17 & $\mathrm{O}+\mathrm{HCO} \longrightarrow \mathrm{CO}_{2}+\mathrm{H}$ & \\
\hline
\end{tabular}

Table 3. Energy barriers against diffusion $\left(E_{\mathrm{b}}\right)$ and desorption energies $\left(E_{\mathrm{D}}\right)(\mathrm{K})$ for selected species.

\begin{tabular}{lrrc|lrrc}
\hline \hline Species & $E_{\mathrm{b}}$ & $E_{\mathrm{D}}$ & Ref. & Species & $E_{\mathrm{b}}$ & $E_{\mathrm{D}}$ & Ref. \\
\hline $\mathrm{H}$ & 100 & 350 & 1 & $\mathrm{C}$ & 240 & 800 & 1 \\
$\mathrm{O}$ & 240 & 800 & 1 & $\mathrm{CH}$ & 196 & 654 & 2 \\
$\mathrm{OH}$ & 378 & 1260 & 2 & $\mathrm{CH}_{2}$ & 287 & 956 & 3 \\
$\mathrm{H}_{2}$ & 135 & 450 & 1 & $\mathrm{CH}_{3}$ & 348 & 1160 & 3 \\
$\mathrm{O}_{2}$ & 363 & 1210 & 3 & $\mathrm{CH}_{4}$ & 408 & 1360 & 3 \\
$\mathrm{H}_{2} \mathrm{O}$ & 558 & 1860 & 3 & $\mathrm{~N}$ & 240 & 800 & 1 \\
$\mathrm{CO}$ & 363 & 1210 & 2 & $\mathrm{NH}$ & 181 & 604 & 3 \\
$\mathrm{HCO}$ & 453 & 1510 & 3 & $\mathrm{NH}_{2}$ & 257 & 856 & 3 \\
$\mathrm{H}_{2} \mathrm{CO}$ & 528 & 1760 & 2 & $\mathrm{NH}_{3}$ & 333 & 1110 & 3 \\
$\mathrm{CH}_{2} \mathrm{OH}$ & 651 & 2170 & 3 & & & & \\
$\mathrm{CH}_{3} \mathrm{OH}$ & 618 & 2060 & 2 & & & & \\
$\mathrm{CO}_{2}$ & 750 & 2500 & 3 & & & & \\
\hline
\end{tabular}

${ }^{1}$ Tielens \& Allamandola (1987).

${ }^{2}$ Allen \& Robinson (1977).

${ }^{3}$ Hasegawa \& Herbst (1993).

of Hasegawa et al. (1992). The use of diffusion rates in both rate equation and master equation treatments is considered in Stantcheva et al. (2002). The barriers and binding energies used here are lower than the M2 models of Ruffle \& Herbst (2000), who based their higher values on new measurements of $\mathrm{H}$ and $\mathrm{H}_{2}$ as analyzed by Katz et al. (1999). Our parameters are the so-called M1 values of Ruffle \& Herbst (2000), which represent low, historical estimates for silicate surfaces or surfaces that are partially icy, except that we allow $\mathrm{H}$ atoms to tunnel from one surface site to another and use a somewhat lower diffusion barrier for $\mathrm{H}$, in the style of Hasegawa et al. (1992). Had we followed the M2 approach outlined in Ruffle \& Herbst (2000) and scaled up the energy barriers of the
Table 4. Rates for selected species at $10 \mathrm{~K}, 15 \mathrm{~K}$, and $20 \mathrm{~K}$.

\begin{tabular}{|c|c|c|c|c|}
\hline $\mathrm{Sp}$ & $\begin{array}{c}k_{\mathrm{acc}}{ }^{1} \\
\left(\mathrm{~cm}^{3} \mathrm{~s}^{-1}\right)\end{array}$ & $\begin{array}{l}t_{\text {thd }}^{-1} 2 \\
\left(\mathrm{~s}^{-1}\right)\end{array}$ & $\begin{array}{l}t_{\mathrm{crd}}^{-13} \\
\left(\mathrm{~s}^{-1}\right)\end{array}$ & $\begin{array}{l}t_{\text {diff }}^{-1} \quad 4 \\
\left(\mathrm{~s}^{-1}\right)\end{array}$ \\
\hline \multicolumn{5}{|c|}{$10 \mathrm{~K}:$} \\
\hline $\mathrm{H}$ & $1.5(-5)$ & $1.9(-3)$ & $6.0(-9)$ & $5.1(4)$ \\
\hline $\mathrm{O}$ & $3.6(-6)$ & $2.0(-23)$ & $3.7(-12)$ & $4.2(-5)$ \\
\hline $\mathrm{C}$ & $4.2(-6)$ & $2.3(-23)$ & $4.2(-12)$ & $4.9(-5)$ \\
\hline $\mathrm{N}$ & $3.9(-6)$ & $2.2(-23)$ & $3.9(-12)$ & $4.5(-5)$ \\
\hline \multicolumn{5}{|c|}{$15 \mathrm{~K}:$} \\
\hline $\mathrm{H}$ & $1.8(-5)$ & $2.2(2)$ & $6.0(-9)$ & $5.5(4)$ \\
\hline $\mathrm{O}$ & $4.4(-6)$ & $7.7(-12)$ & $3.7(-12)$ & $1.3(-1)$ \\
\hline $\mathrm{C}$ & $5.1(-6)$ & $8.9(-12)$ & $4.2(-12)$ & $1.5(-1)$ \\
\hline $\mathrm{N}$ & $4.7(-6)$ & $8.3(-12)$ & $3.9(-12)$ & $1.4(-1)$ \\
\hline \multicolumn{5}{|c|}{$20 \mathrm{~K}:$} \\
\hline $\mathrm{H}$ & $2.0(-5)$ & $7.5(4)$ & $6.0(-9)$ & 7.1(4) \\
\hline $\mathrm{O}$ & $5.1(-6)$ & $4.8(-6)$ & $3.7(-12)$ & 7.1(0) \\
\hline $\mathrm{C}$ & $5.9(-6)$ & $5.5(-6)$ & $4.2(-12)$ & $7.7(0)$ \\
\hline $\mathrm{N}$ & $5.5(-6)$ & $5.1(-6)$ & $3.9(-12)$ & $7.1(0)$ \\
\hline
\end{tabular}

non-hydrogenic surface species, the reactions between the species would have been slowed down sufficiently to cause an increase in the abundances of reactive species on the surface. In this case, a stochastic approach is only important if the grains are very small, thus limiting the number of reactive species to a level at which the stochastic nature of the surface chemistry becomes critical again (Biham et al. 2001; Lipshtat et al. 2004). Since our models contain only spherical grains of uniform radius $0.1 \mu \mathrm{m}$, we have focused our work on the application of the master equation method to surface chemistry in the case of low energy barriers and $\mathrm{H}$ tunneling, resulting in high diffusion rates.

\section{Results}

Rather than using the rather polysyllabic adjectives "stochasticdeterministic" and "purely deterministic" for the two types of models, we will use the descriptive phrases "master equation" and "rate equation", respectively.

\subsection{Probabilistic surface species}

Figures 1 and 2 show computed results at $10 \mathrm{~K}$ for the absolute abundances $\left(\mathrm{cm}^{-3}\right)$ of the probabilistic surface species as functions of time, as calculated by the master equation and rate equation models, respectively. Since the abundances of probabilistic species within certain families are similar, we have made these and subsequents plots more readable by only plotting one species per family. For example, the abundance of $\mathrm{O}$ is close to that of $\mathrm{OH}$, while those for the carbon group of $\mathrm{C}$, $\mathrm{CH}, \mathrm{CH}_{2}$, and $\mathrm{CH}_{3}$ and the nitrogen group of $\mathrm{N}, \mathrm{NH}$, and $\mathrm{NH}_{2}$ 


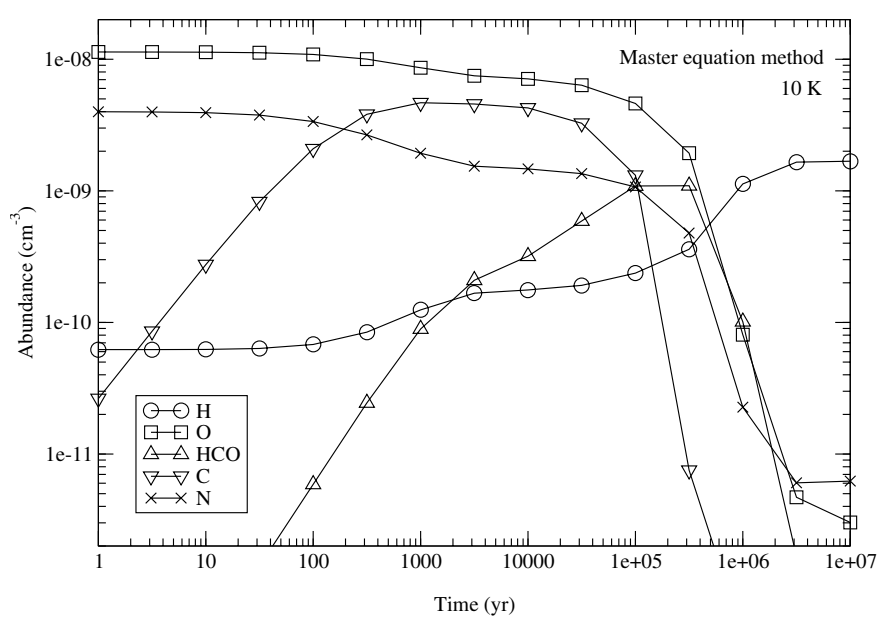

Fig. 1. Absolute abundance of probabilistic surface species vs. time for the master equation method at $10 \mathrm{~K}$.

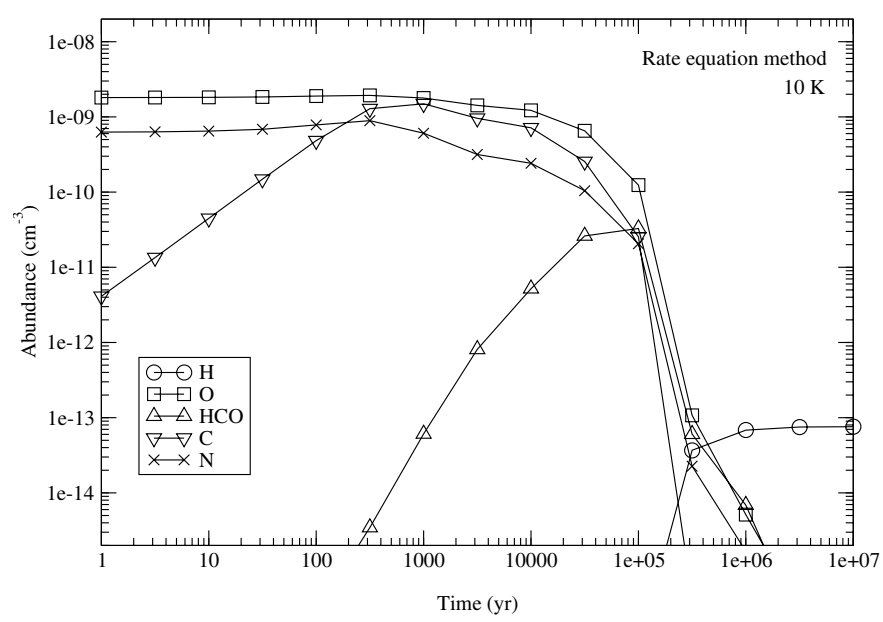

Fig. 2. Absolute abundance of probabilistic surface species vs. time for the rate equation method at $10 \mathrm{~K}$.

show similar to identical abundances among group members. With a grain density $n_{\mathrm{d}}$ of $1.33 \times 10^{-12} n_{\mathrm{H}}$, an absolute abundance of $2.66 \times 10^{-8} \mathrm{~cm}^{-3}$ corresponds to 1 particle per grain. It is easily seen that the number of probabilistic species per grain is never larger than one, so that the accretion limit indeed pertains. Analogous results for the master equation model at temperatures of $15 \mathrm{~K}$ and $20 \mathrm{~K}$ are given in Figs. 3 and 4, where it can also be seen that the accretion limit pertains.

A comparison of the results in Figs. 1 and 2 shows immediately that the abundances of the probabilistic species are far greater with the master equation treatment. The most extreme case is for atomic $\mathrm{H}$, where the difference is many orders of magnitude. At $10^{7} \mathrm{yr}$, the $\mathrm{H}$ abundance in the master equation treatment is on the order of 0.1 species per grain whereas it is approximately $3 \times 10^{-6}$ species per grain with the rate equation method. A detailed understanding of this phenomenon is given in Lipshtat et al. (2004). It must be remembered, however, that the probabilistic species are minor grain components that are not detectable; differences in major grain components between the two methods may be far less.

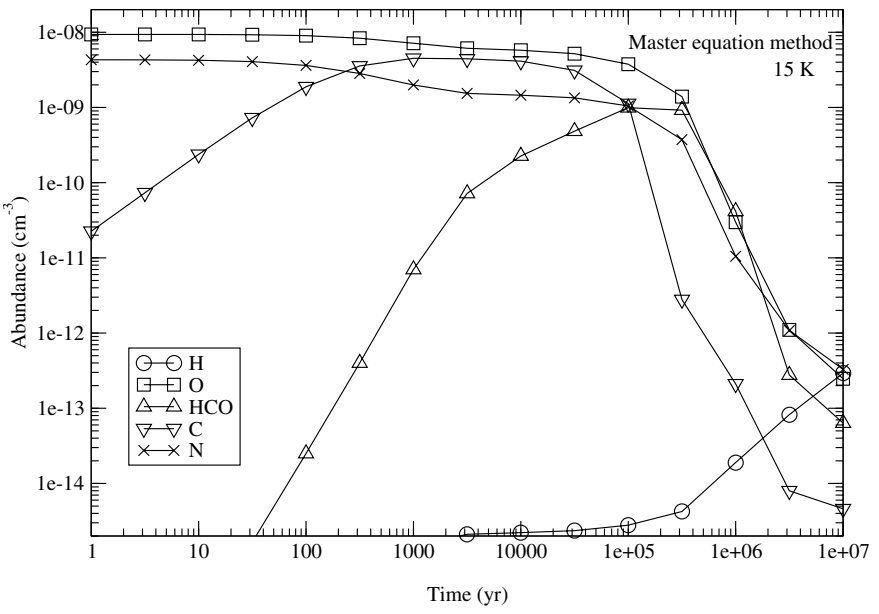

Fig. 3. Absolute abundance of probabilistic surface species vs. time for the master equation method at $15 \mathrm{~K}$.

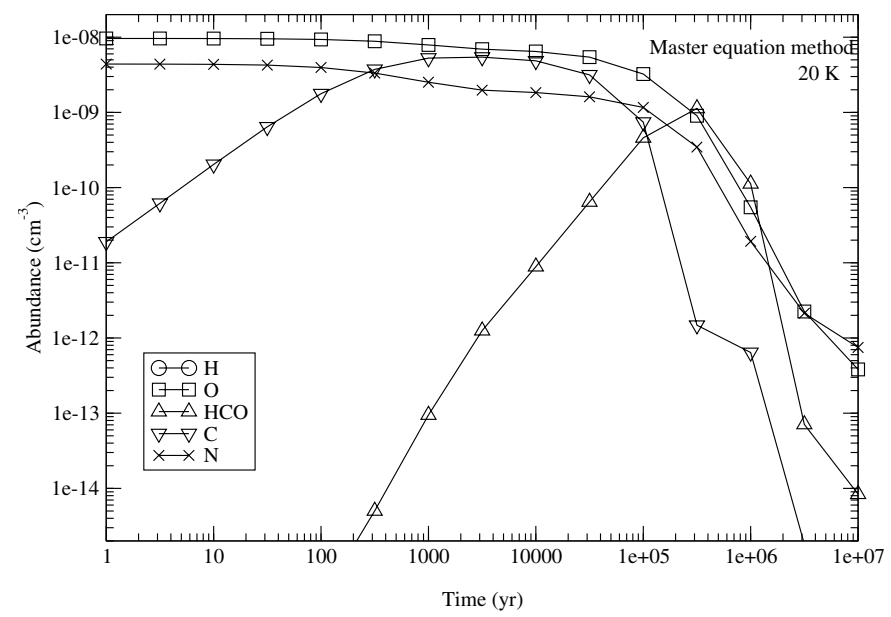

Fig. 4. Absolute abundance of probabilistic surface species vs. time for the master equation method at $20 \mathrm{~K}$.

In addition, although not shown on our plots, the rate equation model produces identical abundances for selected species within the oxygen $(\mathrm{O}, \mathrm{OH})$, carbon $\left(\mathrm{C}, \mathrm{CH}, \mathrm{CH}_{2}\right.$, and $\left.\mathrm{CH}_{3}\right)$ and nitrogen $\left(\mathrm{N}, \mathrm{NH}\right.$, and $\left.\mathrm{NH}_{2}\right)$ groups. This result is reasonable, since a quasi steady-state condition is reached quickly for these species such that on average the production and destruction rates of the species within each of the three groups are equal to the others in the same group. Only an approach that takes into consideration fluctuations can show differences, albeit small. It can also be seen from the figures that a plateau of quasi-constant abundance for most of the probabilistic species is reached within $10-100$ years, and remains present until $10^{5}-10^{6}$ years. At longer times, the gas-phase atomic sources for these probabilistic species tend to dry up as the gas-phase is depleted of heavy species.

At $15 \mathrm{~K}$ and $20 \mathrm{~K}$, there is much better agreement between the rate equation and master equation approaches, as can be seen in Fig. 5 for the case of atomic hydrogen. One reason for the agreement at higher temperatures is that the evaporation rate for atomic hydrogen, the principal reactive species in surface chemistry, becomes comparable with the diffusion 


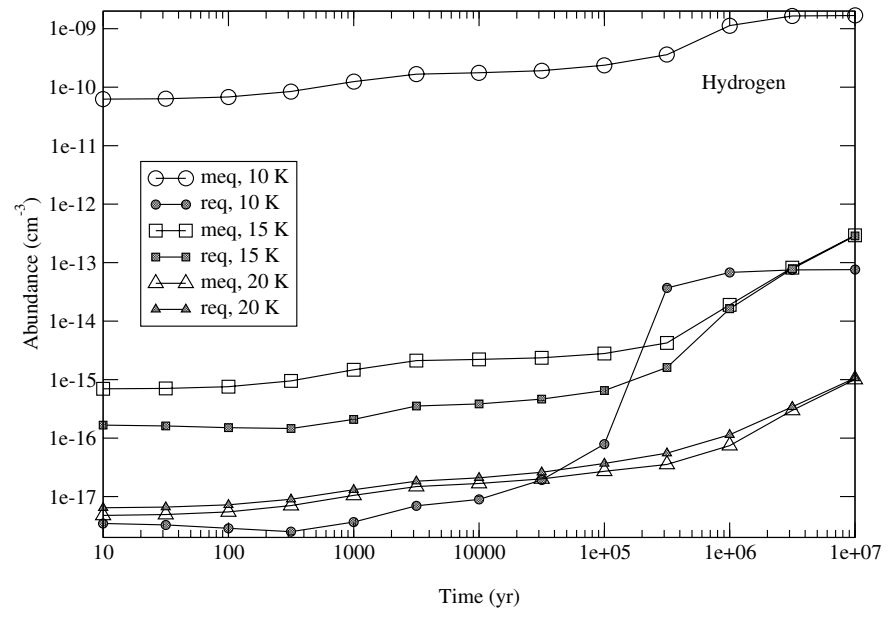

Fig. 5. Absolute abundance for surface $\mathrm{H}$ at $10 \mathrm{~K}, 15 \mathrm{~K}$, and $20 \mathrm{~K}$. The acronyms meq and req stand, respectively, for master equation and rate equation.

rate. Under these conditions, although the surface abundance of $\mathrm{H}$ is quite low, the dominant destructive term is no longer overwhelmingly the reactive one, and the evaporative process is well-represented by the simple exponential decay of the rate equation approach. A more detailed analysis of this effect can be found in Biham et al. (2001) and Lipshtat et al. (2004).

It is also of interest to see if the modified rate equation method (Caselli et al. 1998, 2002; Stantcheva et al. 2001; Ruffle $\&$ Herbst 2000) proves to be an adequate substitute for the master equation approach. The modified rate method utilized here follows the approach of Caselli et al. (2002) except for reactions with activation energy, where the rate coefficient for reaction, which includes a tunneling factor, is slowed to the faster of the accretion and evaporation rates of the atomic reactant if necessary. In the treatment of Caselli et al. (2002), a somewhat more complex algorithm is used, in which one considers the product of the rate coefficient and the abundant surface species depleted by the reaction. The greater complexity of the Caselli et al. approach renders it difficult to extend to a large model such as considered here. For the probabilistic species, the calculated abundances obtained with our approach are in good to excellent agreement with that obtained from the master equation method. In general, the abundances of the modified rate method tend to be somewhat higher but still accurate to within a factor of a few.

\subsection{Gas-phase and stable surface species}

Figure 6 shows plots of fractional abundances vs. time for the stable gas-phase species water, carbon monoxyde, hydrogen cyanide, and ammonia, obtained from both the master equation and rate equation methods at $10 \mathrm{~K}$. The agreement between the two models is almost complete until times of $10^{6}$ years; after that, there is a slight difference due to accretion, which becomes appreciable, and surface chemistry.

Figure 7 shows calculated results for the fractional abundances of molecular oxygen, water, methane and ammonia (with respect to $\mathrm{H}_{2}$ ) on grain surfaces at $10 \mathrm{~K}$, as calculated

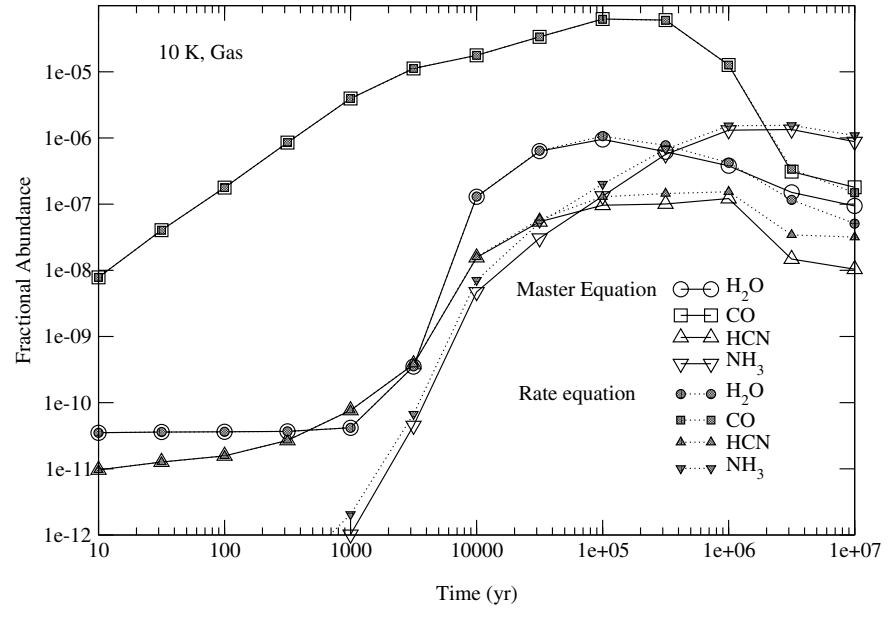

Fig. 6. Fractional abundances of assorted gas-phase species (relative to $\mathrm{H}_{2}$ ) vs. time at $10 \mathrm{~K}$ obtained from the master equation and rate equation methods.

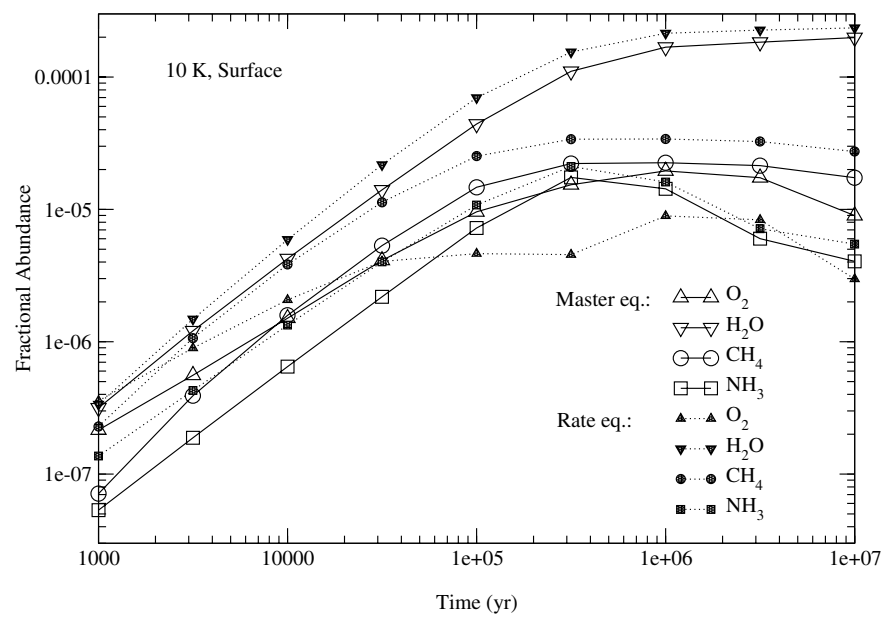

Fig. 7. Evolution of surface fractional abundances (relative to $\mathrm{H}_{2}$ ) for stable species at $10 \mathrm{~K}$, as calculated by master equation and rate equation methods.

by both the master equation and rate equation approaches. While there are discrepancies as large as a factor of 5, at later times $\left(10^{6}-10^{7} \mathrm{yr}\right)$ the results typically converge to within a factor of a few. If one looks at Fig. 8, which contains analogous results for the species $\mathrm{CO}, \mathrm{CO}_{2}, \mathrm{H}_{2} \mathrm{CO}$, and $\mathrm{CH}_{3} \mathrm{OH}$, one sees larger divergences. A major discrepancy concerns the production of methanol from CO. In the stochastic model, all CO molecules appear to be converted almost immediately to methanol, while in the deterministic model the conversion is more leisurely, and essentially requires a monolayer of CO molecules, or a fractional abundance of $10^{-6}$ to become efficient. The leisurely pace arises from the low average surface abundance of atomic $\mathrm{H}$ and the inefficiency of the $\mathrm{H}+\mathrm{CO}$ reaction, which possesses a barrier. When this critical abundance is reached at $\approx 10^{5} \mathrm{yr}$, methanol becomes the most abundant carbon-containing molecule on the surface in agreement with the master equation model. The two models are never in agreement for $\mathrm{CO}$ and $\mathrm{H}_{2} \mathrm{CO}$, whereas for $\mathrm{CO}_{2}$, a large early-time discrepancy is erased at late times. 


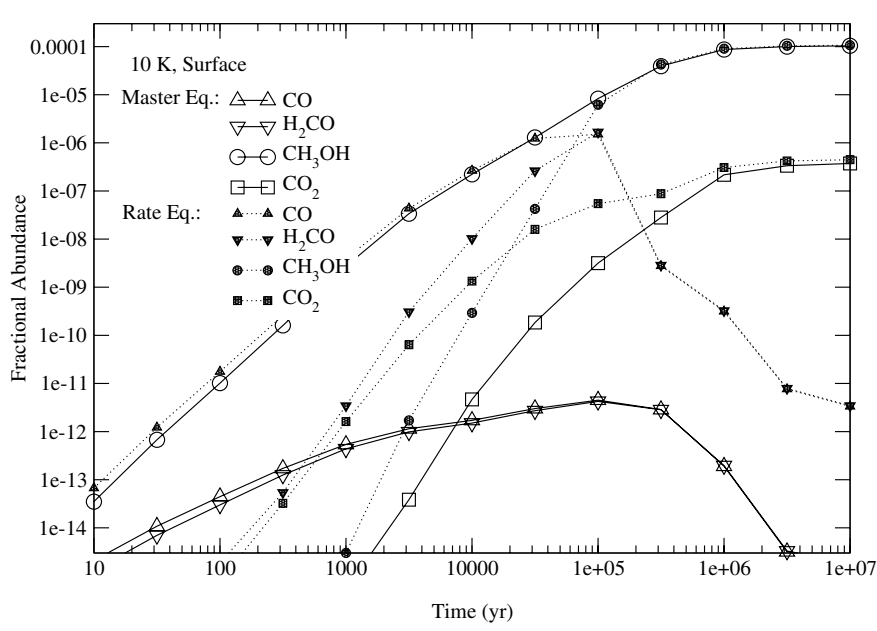

Fig. 8. Evolution of surface fractional abundances (relative to $\mathrm{H}_{2}$ ) for additional stable species at $10 \mathrm{~K}$ as calculated by master equation and rate equation methods.

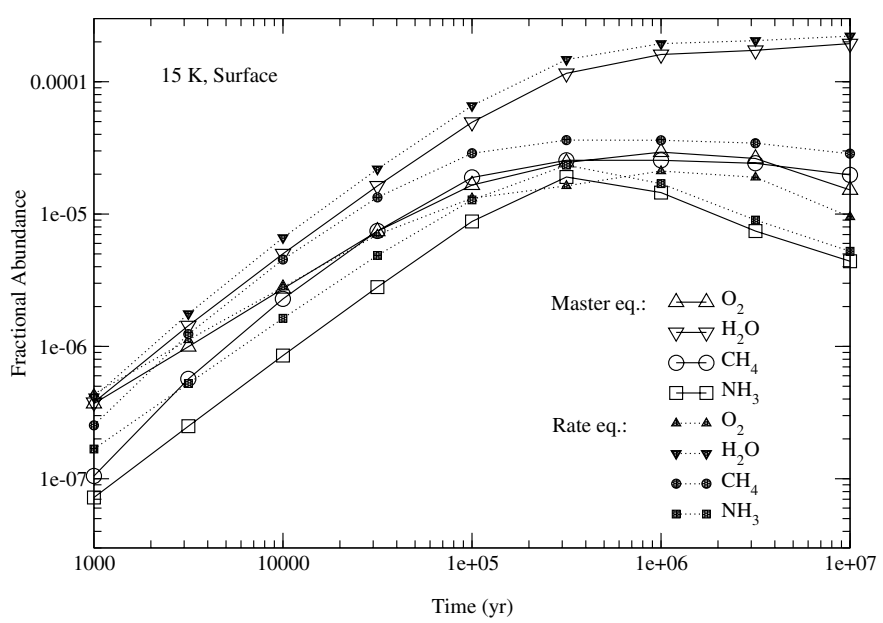

Fig. 9. Evolution of surface fractional abundances (relative to $\mathrm{H}_{2}$ ) for stable species at $15 \mathrm{~K}$ as calculated by master equation and rate equation methods.

Results for the master equation and rate equation models at $15 \mathrm{~K}$ are shown in Figs. 9 and 10 while results at $20 \mathrm{~K}$ are depicted in Figs. 11 and 12, respectively. The agreement between the two models appears to be best at $15 \mathrm{~K}$, and it deteriorates at still higher temperatures. At $20 \mathrm{~K}$, the water produced by the rate equation model is almost two orders of magnitude less than that in the stochastic model for long periods of time, and only at $10^{7} \mathrm{yr}$ do the results of both models converge to within a factor of a few. The discrepancy for $\mathrm{CO}_{2}$ is even worse; the abundance of surface $\mathrm{CO}_{2}$ is more than a hundred times lower in the master equation model than in the rate equation approach at all times. The results for the remaining molecules are in fair agreement, even for $\mathrm{CO}$ and methanol at early times. It is clear that the rate equation method cannot be counted on to always be reliable for surface abundances for the situations studied in this paper.

The results for the modified rate method at $10 \mathrm{~K}, 15 \mathrm{~K}$, and $20 \mathrm{~K}$ are generally closer to the master equation approach than are the results of the standard rate equation approach. At $10 \mathrm{~K}$, the only signficant problem is $\mathrm{CO}_{2}$, for which the modified rate

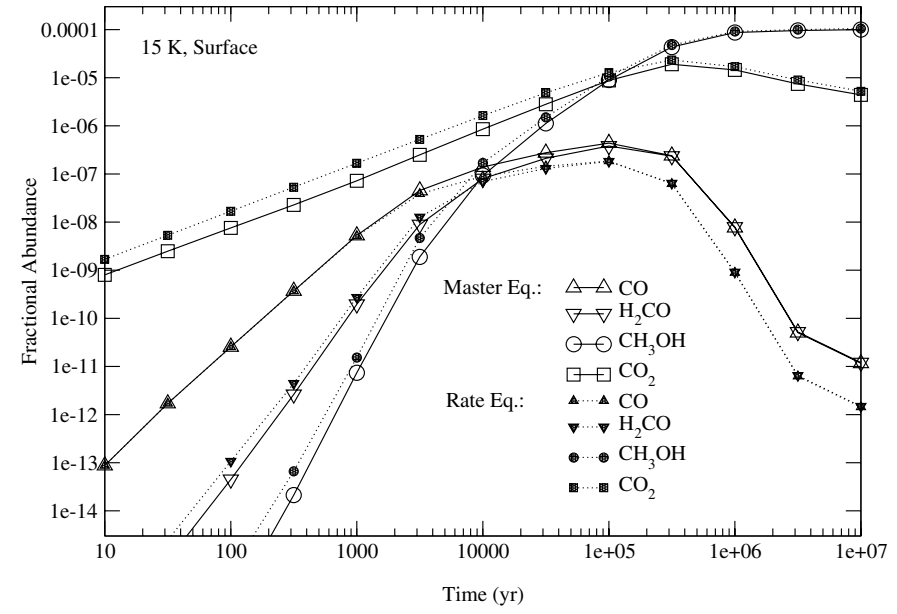

Fig. 10. Evolution of surface fractional abundances (relative to $\mathrm{H}_{2}$ ) for additional stable species at $15 \mathrm{~K}$ as calculated by master equation and rate equation methods.

approach yields far too large an abundance except at late times. This problem lessens at higher temperatures, where the agreement is fine for most other species, except for $\mathrm{O}_{2}$, which is overproduced at $20 \mathrm{~K}$ by factors of 5-10. The carbon monoxide to methanol conversion is handled exactly at all three temperatures. We conclude that if one cannot use the stochastic approach in a given model, the modified rate approach is a better alternative than its unmodified counterpart. In fact, it might be useful to look in some detail at the reason for the discrepancies between our modified rate approach and the stochastic treatment in an attempt to improve the former.

\section{Comparison with observations and other models}

Although the main purpose of this paper is to introduce the mixed stochastic-deterministic approach to gas-grain chemistry, it is also of interest to compare our results with observations of grain mantles. In this comparison, one must keep in mind that our approach, though close to mathematically exact, is based on a slew of simplifying assumptions about grains: (i) the grains all have the same size; (ii) the grains are homogeneous; (iii) the only reaction mechanism of importance is the diffusive (Langmuir-Hinshelwood) one; (iv) all species in mantles can react no matter what monolayer they reside in; and (v) desorption occurs mainly via cosmic-ray induced heating. Moreover, a set of barriers against diffusion and desorption was chosen not because they are likely to be the most accurate ones, but because they lead to the largest possible discrepancies between stochastic and deterministic approaches. Finally, the activation energy barriers for some important reactions (e.g. $\mathrm{H}+\mathrm{CO}$ ) are highly uncertain. Thus, any differences between model results and observation do not vitiate the use of the stochastic approach, but call into question the parameters and simplifications chosen. Master equation models with a distribution of grain sizes have already been calculated for the simple case of $\mathrm{H}_{2}$ and $\mathrm{HD}$ formation (Lipshtat et al. 2004), and we are currently exploring the role of grain inhomogeneities. 
Table 5. Comparison with observations.

\begin{tabular}{lrrrrrrr}
\hline \hline Species & RAFGL & W33A $^{b, c}$ & Elias $16 c^{c}$ & \multicolumn{2}{c}{ Master equation, $10 \mathrm{~K}$} & \multicolumn{2}{c}{ Master equation, 20 K } \\
& $7009 \mathrm{~S}^{a, b}$ & & & $3.2 \times 10^{5}$ years & $10^{6}$ years & $3.2 \times 10^{5}$ years & $10^{6}$ years \\
\hline $\mathrm{H}_{2} \mathrm{O}$ & $2.1(-4)$ & $1.1(-4)$ & $1.25(-4)$ & $1.1(-4)$ & $1.7(-4)$ & $1.1(-4)$ & $1.4(-4)$ \\
$\mathrm{CO}$ & 16.4 & 8 & 25 & $2.6(-6)$ & $1.1(-7)$ & 30 & 1.9 \\
$\mathrm{CO}_{2}$ & 22.7 & 13 & 18 & 0.025 & 0.13 & 0.05 & 0.17 \\
$\mathrm{CH}_{3} \mathrm{OH}$ & 32.3 & 18 & $<3$ & 35.7 & 51.8 & 13 & 72 \\
$\mathrm{NH}_{3}$ & - & 15 & $<9$ & 15.8 & 8.5 & 0.05 & 0.05 \\
$\mathrm{CH}_{4}$ & 3.9 & 1.5 & - & 20.1 & 13.4 & 1.4 & 1.4 \\
\hline
\end{tabular}

${ }^{a}$ D'Hendecourt et al. (1996).

${ }^{b}$ Dartois et al. (1999).

${ }^{c}$ For references, see Gibb et al. (2000).

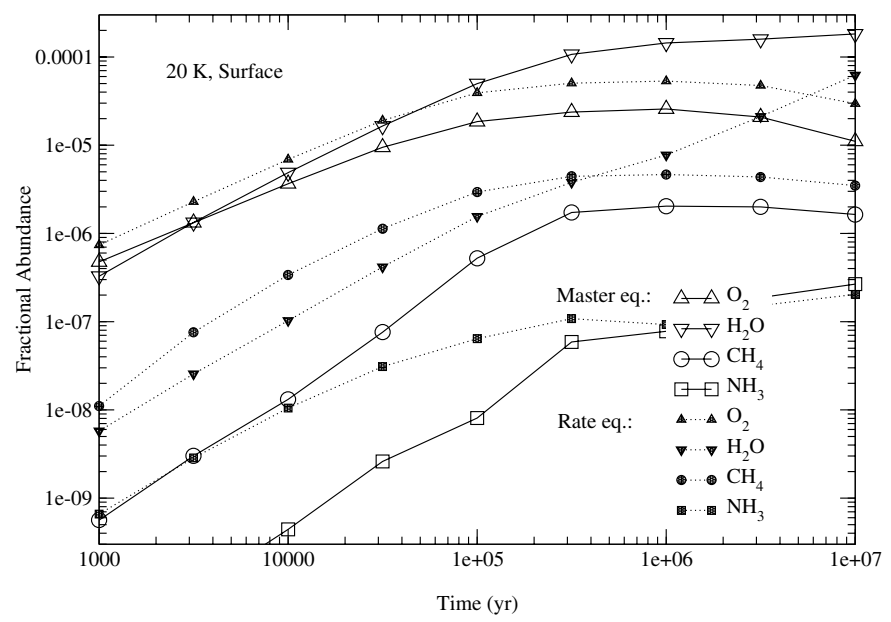

Fig. 11. Evolution of surface fractional abundances (relative to $\mathrm{H}_{2}$ ) for stable species at $20 \mathrm{~K}$ as calculated by master equation and rate equation methods.

In Table 5, we compare results at $10 \mathrm{~K}$ and $20 \mathrm{~K}$ with observations of grain mantle ices towards the quiescent source in front of the field star Elias 16 (Whittet et al. 1998; Chiar et al. 1995, 1996) and towards the high-mass protostellar objects RAFGL7009S and W33A (D'Hendecourt et al. 1996; Dartois et al. 1999; Gibb et al. 2000). The results for water ice are given as fractions of the total hydrogen density, whereas the remaining molecular abundances are shown as percentages of the amount of water ice. The major difference between Elias 16 and the material in front of the high-mass protostellar objects is the larger amount of methanol ice in the latter objects. We compare the observed results with calculated values for $3.2 \times 10^{5}$ and $10^{6} \mathrm{yr}$, when the amount of water in our model becomes comparable with that in the observed sources. The agreement between the theoretical predictions and the observational data is mixed. For Elias 16, a temperature of $10 \mathrm{~K}$ would appear to be the more physically reasonable. Our results at $10 \mathrm{~K}$ show virtually no solid $\mathrm{CO}$ and little solid $\mathrm{CO}_{2}$, in strong contradiction with observation. The model appears to be very efficient in production of methanol, but there is little methanol to be found in Elias 16. For a dust temperature of $20 \mathrm{~K}$ and a time of $3.2 \times 10^{5} \mathrm{yr}$, a reasonable amount of $\mathrm{CO}$ is predicted, but the amount of $\mathrm{CO}_{2}$ is still under-predicted and methanol still

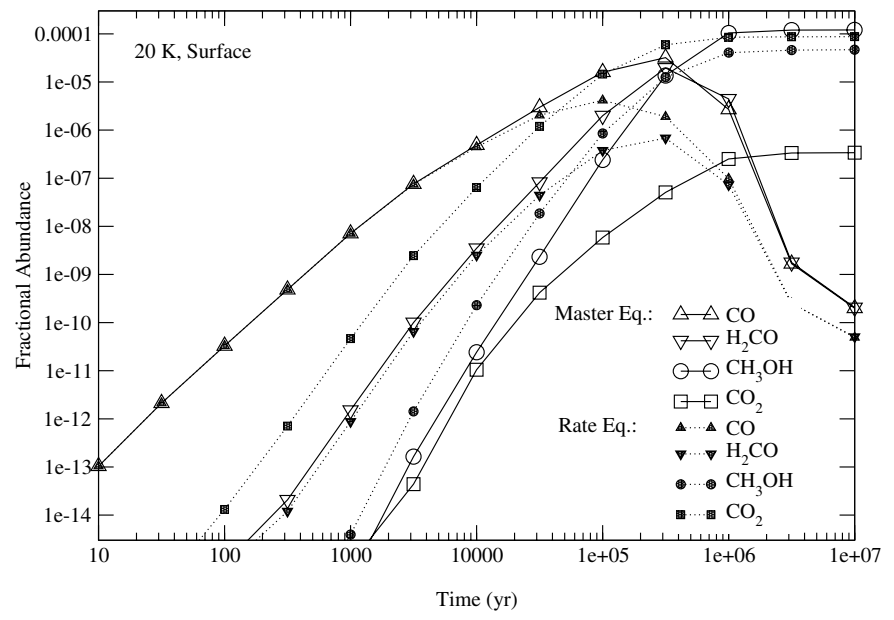

Fig. 12. Evolution of surface fractional abundances (relative to $\mathrm{H}_{2}$ ) for additional stable species at $20 \mathrm{~K}$ as calculated by master equation and rate equation methods.

over-predicted, if not as badly so. Thus, model and observation are in poor agreement.

For the two sources seen against protostellar objects, our results at $20 \mathrm{~K}$ are reasonable for water, $\mathrm{CO}$, methanol, and methane ices, while our results at $10 \mathrm{~K}$ are reasonable for water, methanol, and ammonia ices. Neither temperature works well for the production of $\mathrm{CO}_{2}$ ice. The mixed pattern of agreement and disagreement is not surprising given the number of assumptions made. In addition, a better knowledge of physical conditions in each source would appear to be necessary to further progress. It may be futile to model these sources, especially those in front of massive protostars, as homogeneous systems.

Pontoppidan et al. (2003) have recently reported the first detection of large abundances of methanol ice in low mass protostars. In a large survey of approximately 40 low mass young stellar objects, they detected abundant methanol in three of them. Two of the sources are part of the dense cluster SVS4 in the Serpens cloud core and the third source is Cha INa 2 in the Chameleon I cloud. The fraction of methanol abundance (with respect to solid water) is reported as $21 \%, 25 \%$, and $14 \%$ in SVS4-5, SVS4-9, and Cha INa2, respectively. These values lie within the range of predicted methanol abundances in our 
models. Yet, until the physical conditions in these objects are properly understood, we cannot determine with certainty how well our predictions agree with the observations.

One set of parameters that can be easily varied is the set of diffusion and desorption energies. With an assortment of values for these, and with the exclusion of $\mathrm{H}$ tunneling, Ruffle \& Herbst (2000) ran gas-grain models to study Elias 16, which they also considered to be a homogeneous source. With a much more complete network of surface reactions, they were able to reproduce the $\mathrm{CO}$ abundance and upper limit to the methanol abundance using a model (M2/A) in which diffusion rates are much lower than those used here. None of their models, however, produces a sufficient amount of $\mathrm{CO}_{2}$ ice. In a subsequent paper, Ruffle \& Herbst (2001) showed that they could produce a sufficient amount of this ice as well as reproduce other observed abundances by using lower energy barriers against diffusion (the M1 conditions), and raising the temperature to $12 \mathrm{~K}$, or increasing the density by two orders of magnitude. A simplified modified rate method was used in these calculations, which would appear to be perfectly adequate with the parameters chosen. Thus, there is some evidence that our diffusion and desorption energies, especially those for the all-important reactive species atomic hydrogen, are too low. Possibly our assumption of $\mathrm{H}$ tunneling is also incorrect.

\section{Discussion}

We have presented a mixed stochastic-deterministic gas-grain model to study the chemistry of cold interstellar cloud cores. In our approach, the gas-phase chemistry is simulated via deterministic rate equations while the diffusive surface chemistry is handled stochastically via direct integration of the chemical master equation. The model can run on current supercomputer technology within a reasonable amount of time. The results are compared with purely deterministic models, in which the rate equation approach is utilized to simulate both gas and surface chemistry. We have studied the chemistry occurring in cold interstellar cloud cores at $10 \mathrm{~K}, 15 \mathrm{~K}$, and $20 \mathrm{~K}$, and have utilized low values for surface diffusion barriers (Hasegawa et al. 1992) so as to make the need for a stochastic approach most acute.

Comparison between the calculated abundances of the master equation and rate equation models shows a mixed pattern. For gas-phase species, the differences are small, even at times as large as $10^{7} \mathrm{yr}$, when surface adsorption and desorption effects are important. For surface species, the differences can be much larger. We find that the rate equation model can underestimate the abundances of reactive granular species by orders of magnitude compared with the master equation model. In both models, however, these abundances correspond to fewer than one particle per grain, thus confirming that the system is in the accretion limit, a limit in which stochastic methods are required if the small abundances are due to reactive processes. Unfortunately, since the abundances are too low for the species to be detected, there are no observations against which these predictions can be compared. The differences in the abundances of the stable surface molecules tend to become less striking as time evolves, so that by $10^{5} \mathrm{yr}$, discrepancies are generally less than a factor of five, with some notable exceptions. At $10 \mathrm{~K}$, the conversion of surface $\mathrm{CO}$ into formaldehyde and then methanol occurs far more rapidly with the master equation method. This process includes two reactions with small activation energy barriers $(\mathrm{H}+\mathrm{CO}, \mathrm{H}+$ $\mathrm{H}_{2} \mathrm{CO}$ ), a situation in which the stochastic method shows much greater efficiency. At $15 \mathrm{~K}$, the difference in efficiency has all but disappeared, and the agreement between models is excellent. But, by $20 \mathrm{~K}$, severe disagreements reemerge for most times, especially involving the molecules water and $\mathrm{CO}_{2}$. Here the rate equation model has great difficulty in the production of water ice, presumably because of the short evaporation time for surface hydrogen atoms. The discrepancies are less for ammonia and methane ices because of the gas-phase synthetic routes. On the other hand, the master equation model has far more difficulty in the production of surface $\mathrm{CO}_{2}$. We conclude that in the accretion limit, where reactive surface species have average abundances of less than unity per grain due to rapid reaction, error can be introduced by the use of deterministic rate equations in handling the surface chemistry. Contrary to this negative assessment, the modified rate method presents an attractive alternative since it is generally in much better agreement with the stochastic approach to surface chemistry. There are still discrepancies, however, so that, if possible, the stochastic method should be used in the accretion limit unless the modified rate approach can be further improved for large networks.

In comparing our results, as well as the results of previous gas-grain models, with observation, one must confront the fact that the physical conditions in the sources with analyzed surface spectra are not well understood. Sources lying in front of infrared-emitting massive star formation regions are probably not homogeneous and should perhaps be treated as PDR's. The best studied source illuminated by a background field star Elias 16 - may well be more homogeneous, but its physical conditions have not been studied to the best of our knowledge. With the assumption of a homogeneous gas density of $10^{4} \mathrm{~cm}^{-3}$ and a temperature in the range $10-20 \mathrm{~K}$, we have obtained results that are not in consistent agreement with observed surface abundances in any of the three sources considered (RAFGL7009S and W33A, in addition to Elias 16). There are of course many sources of uncertainty in our model results, all of which would be problematical even if the exact physical conditions of the sources modeled were well-known.

If one assumes that our major assumptions are correct, and that the physical conditions in the sources are not too different from what we assume, then specific changes in surface reaction rates might be looked at to improve the agreement with observation. One major discrepancy with theoretical data, concerning the too rapid conversion of $\mathrm{CO}$ into methanol at $10 \mathrm{~K}$, would be reduced if the rate of the reactions were slowed down, either by higher diffusion barriers or by larger activation energies of individual reactions. Slowing down the rate of $\mathrm{H}+\mathrm{CO}$ would aid in the production of $\mathrm{CO}_{2}$, a species of which we produce too little at all temperatures studied, by allowing the $\mathrm{CO}$ to react with $\mathrm{O}$. Alternatively, the discrepancy could be reduced by reducing the activation energy barrier for the reaction between $\mathrm{CO}$ and $\mathrm{O}$. The current state of laboratory and theoretical work on these systems is still somewhat confusing and needs 
Table A.1. Percentage difference between initial and final elemental abundances $\left(T=10 \mathrm{~K} ; n\left(\mathrm{H}_{2}\right)=10^{4} \mathrm{~cm}^{-3}\right)$.

\begin{tabular}{lrrrr}
\hline \hline \multirow{2}{*}{ Element } & \multirow{2}{*}{ Rate eq. } & \multicolumn{4}{c}{ Master eq. model } \\
& & 3.00 & 3.01 & 4.01 \\
\hline $\mathrm{H}$ & $6.8(-8)$ & $-2.9(-3)$ & $-2.9(-3)$ & $-1.4(-3)$ \\
$\mathrm{C}$ & $-3.3(-8)$ & -15 & -15 & -11 \\
$\mathrm{~N}$ & $-3.2(-6)$ & -22 & -22 & -12 \\
$\mathrm{O}$ & $-1.6(-6)$ & -14 & -14 & -7.4 \\
\hline
\end{tabular}

to be further refined (Watanabe \& Kouchi 2002; Hiraoka et al. 2000; Roser et al. 2001).

Finally, we have ignored the role of deuterium in surface and gas-phase chemistry in this model, but fractionation can be so large an effect that it can even influence some calculated results of non-deuterated species, albeit typically at densities greater than studied here. The effect of deuterium in models of pure surface chemistry has been studied stochastically (Stantcheva \& Herbst 2002). We are currently developing two gas-grain models with deuterium chemistry; both of these utilize the modified rate method for surface processes. In the first approach, we consider a static model but include a distribution of grain sizes, whereas in the second, we are including collapse to form a pre-stellar core.

Acknowledgements. The Astrochemistry Program at The Ohio State University is supported by The National Science Foundation (US). We thank the Ohio Supercomputer Center for time on their Cray SV1 machine.

\section{Appendix A: A sensitive check for numerical errors}

As another criterion for estimating the numerical error in our calculations, we have kept track of the elemental abundances and have compared their values at the beginning and the end of a calculation. Theoretically, this difference should be zero, if no cut-off (or other unforeseen) error exists. We have tabulated the percentage changes in elemental abundances for master equation models with a variety of cut-off values for probabilistic species. Model 3.00 refers to minimal cutoff values of unity except for $\mathrm{H}, \mathrm{O}, \mathrm{N}$, and $\mathrm{C}$, for which the values are set to two, while the total limit for the set of probabilistic species is three. Model 3.01 refers to a set of individual cut-off values all equal to two except for $\mathrm{HCO}$ and $\mathrm{H}_{3} \mathrm{CO}$, for which the limits are equal to 1 . The total limit is once again three. Model 4.01, used to obtain the results reported in this paper, contains the same set of individual cutoffs as in 3.01, but with a total limit of four. One expects the percentage elemental differences between initial and final values to decrease as one goes to higher cutoffs.

Table A.1 gives percentage elemental differences for Models 3.00, 3.01, and 4.01 as well as for the rate equation model for a temperature of $10 \mathrm{~K}$ and a molecular hydrogen density of $10^{4} \mathrm{~cm}^{-3}$. It can be seen that while the $\mathrm{H}$ elemental
Table A.2. Percentage difference between initial and final elemental abundances.

\begin{tabular}{lrrrr}
\hline \hline Species & \multicolumn{4}{c}{ Master eq. model 3.00 } \\
$T$ & $10 \mathrm{~K}$ & $15 \mathrm{~K}$ & $20 \mathrm{~K}$ & $10 \mathrm{~K}$ \\
$n\left(\mathrm{H}_{2}\right)$ & $10^{4} \mathrm{~cm}^{-3}$ & $10^{4} \mathrm{~cm}^{-3}$ & $10^{4} \mathrm{~cm}^{-3}$ & $10^{5} \mathrm{~cm}^{-3}$ \\
\hline $\mathrm{H}$ & $-2.9(-3)$ & $-2.5(-3)$ & $-1.7(-3)$ & $-7.4(-3)$ \\
$\mathrm{C}$ & -15 & -16 & -12 & -46 \\
$\mathrm{~N}$ & -22 & -20 & -8.5 & -72 \\
$\mathrm{O}$ & -14 & -12 & -11 & -49 \\
\hline
\end{tabular}

abundance seems to be constant to a high degree of certainty, there is a significant numerical error in the $\mathrm{C}, \mathrm{N}$, and $\mathrm{O}$ elemental abundances for the master equation models. As expected, the error is least for the model with the highest cutoffs. Also, as can be seen in Table A.2, the error decreases for the basic model 3.00 as the temperature is raised but increases at a fixed temperature as the gas density is increased. These trends are explained by the fact that the larger the surface abundance of the probabilistic species, the higher the cut-off values required, and therefore, the larger the error when lower cutoffs are used. For example, in the case of $n\left(\mathrm{H}_{2}\right)=10^{5} \mathrm{~cm}^{-3}$, the average surface abundances of atomic $\mathrm{C}, \mathrm{N}$, and $\mathrm{O}$ become larger than one, and we can see from Table A.2 that one-half or more of elemental oxygen, carbon and nitrogen is lost due to cut-off error. In such a case, either higher cutoffs must be considered, or the offending species must be removed from the set of probabilistic species and treated as normal. The loss in elemental abundances can be used as a quick indicator of the accuracy of a master equation model, while multiple runs and comparison of their results will yield information about the actual error in the individual abundances.

\section{References}

Allen, M., \& Robinson, G. W. 1977, ApJ, 212, 396

Bettens, R. P. A., Lee, H.-H., \& Herbst, E. 1995, ApJ, 443, 664

Biham, O., Furman, I., Pirronello, V., \& Vidali, G. 2001, ApJ, 553, 595

Caselli, P., Hasegawa, T. I., \& Herbst, E. 1998, ApJ, 495, 309

Caselli, P., Stantcheva, T., Shalabiea, O., Shematovich, V., \& Herbst, E. 2002, Plan. Sp. Sci., 50, 1257

Cazaux, S., \& Tielens, A. G. G. M. 2002, ApJ, 240, L29

Charnley, S. B. 1998, ApJ, 509, L121

Charnley, S. B. 2001, ApJ, 375, L99

Charnley, S. B., Tielens, A. G. G. M., \& Rodgers, S. D. 1997, ApJ, 482, L203

Chiar, J. E., Adamson, A. J., Kerr, T. H., \& Whittet, D. C. B. 1995, ApJ, 455, 234

Chiar, J. E., Adamson, A. J., \& Whittet, D. C. B. 1996, ApJ, 472, 665

Dartois, E., Schutte, W., Geballe, T. R., et al. 1999, A\&A, 342, L32

D'Hendecourt, L., de Muizon, M. J., Dartois, E., et al. 1996, A\&A, $315, \mathrm{~L} 365$

Gibb, E. L., Whittet, D. C. B., Schutte, W. A., et al. 2000, ApJ, 536, 347

Gillespie, D. 1976, J. Comput. Phys., 22, 403

Gillespie, D. 1992, Physica A, 188, 404 
Green, N. J. B., Toniazzo, T., Pilling, M. J., et al. 2001, A\&A, 375, 1111

Hasegawa, T. I., \& Herbst, E. 1993, MNRAS, 261, 83

Hasegawa, T. I., Herbst, E., \& Leung, C. M. 1992, ApJS, 82, 167

Herbst, E., \& Klemperer, W. 1973, ApJ, 185, 505

Hiraoka, K., Sato, T., \& Takayama, T. 2000, in Astrochemistry: From molecular clouds to planetary systems, ed. Y. C. Minh, \& E. F. van Dishoeck (Chelsea, Michigan: Sheridan Books), 283

Katz, N., Furman, I., Biham, O., Pirronello, V., \& Vidali, G. 1999, ApJ, 522, 305

Lipshtat, A., Biham, O., \& Herbst, E. 2004, MNRAS, 348, 1055

Pickles, J. B., \& Williams, D. A. 1977, Ap\&SS, 52, 443

Pontoppidan, K. M., Dartois, E., van Dishoeck, E. F., Thi, W.-F., \& d'Hendecourt, L. 2003, A\&A, 404, L17

Prasad, S. S., \& Huntress, W. T. 1980, ApJS, 43, 1

Roberts, H., Herbst, E., \& Millar, T. 2002, MNRAS, 336, 283
Roser, J. E., Vidali, G., Manico, G., \& Pirronello, V. 2001, ApJ, 555, L61

Ruffle, D. P., \& Herbst, E. 2000, MNRAS, 319, 837

Ruffle, D. P., \& Herbst, E. 2001, MNRAS, 324, 1054

Stantcheva, T., \& Herbst, E. 2002, MNRAS, 340, 983

Stantcheva, T., Caselli, P., \& Herbst, E. 2001, A\&A, 375, 673

Stantcheva, T., Shematovich, V., \& Herbst, E. 2002, A\&A, 391, 1069

Terzieva, R., \& Herbst, E. 1998, ApJ, 501, 207

Tielens, A. G. G. M., \& Allamandola, L. J. 1987, in Interstellar Processes, ed. D. J. Hollenbach, \& J. H. A. Thronson (Dordrecht), 397

Tielens, A. G. G. M., \& Hagen, W. 1982, A\&A, 114, 245

Watanabe, N., \& Kouchi, A. 2002, ApJ, 571, L173

Whittet, D. C. B., Gerakines, P. A., Tielens, A. G. G. M., et al. 1998, ApJ, 498, 159 